\title{
Researches on the Space-Time View of the Special Relativity
}

\author{
Jidong Zhu \\ Shanghai University of Electric Power, Shanghai, China \\ Email: zhu_jidong@126.com
}

How to cite this paper: Zhu, J.D. (2017) Researches on the Space-Time View of the Special Relativity. Open Access Library Journal, 4: e3334.

http://dx.doi.org/10.4236/oalib.1103334

Received: January 3, 2017

Accepted: January 17, 2017

Published: January 20, 2017

Copyright () 2017 by author and Open Access Library Inc.

This work is licensed under the Creative Commons Attribution International License (CC BY 4.0).

http://creativecommons.org/licenses/by/4.0/ (c) (7) Open Access

\begin{abstract}
For the last one hundred years, researches on Special Relativity have always been lively. Experiments concerned have been recorded in many articles. After a lot of reading, analysis of these articles and information, the author found that some classical experiments have indicated that the time dilation has not symmetric property, so the space-time view of the special relativity is wrong. According to the special relativity, observers can only observe the rhythm of motion clock dropping off, but in the experiments, people can observe not only the rhythm of motion clock dropping off, but also such two phenomena: the rhythm of motion clock going up, and the rhythm of motion clock unchanged. The rhythm of motion clock dropping off has been called the time dilation effect by people; here the author can only call the "phenomenon of the rhythm of motion clock going up" time contraction effect, and "the phenomenon of the rhythm of motion clock unchanged" time equivalence effect. Because the special relativity cannot explain the three effects of time dilation, time contraction and time equivalence, therefore the author has reinterpreted these experiments with a new point of view, and has solved the contradiction between the space-time theory and the experiments.
\end{abstract}

\section{Subject Areas}

Special Theory of Relativity

\section{Keywords}

Special Relativity, Space-Time View, Time Dilation, Doppler Shift

\section{Preface}

To explain the zero conclusion of Michelson-Morley experiment, Lorentz and some physicists raised the conception of time dilation and space contraction. 
They considered that the ether exists, and space contraction and time dilation exist only in the inertia reference frame which has relative motion with ether [1]. Einstein gave up the view of ether in his Special Relativity and gave out new explanations to the physical meaning of Lorentz Transformation. Einstein considered any inertia frame as the same right, for example, two inertia frames $k$ and $k^{\prime}$ which have relative motion with each other. All the observers in both $k$ and $k^{\prime}$ think that they rest and others move. Time dilation is relative. The observers in $k$ and $k^{\prime}$ all put countless clocks in their own frame and compare them with a certain clock in another inertia frame. These observers in $k$ and $k^{\prime}$ will find that "clock" in the other reference frames is slow [2].

There were two different views of Space-Time in physics field-Lorentz's absolute and Einstein's relative view. The former used ether to explain the absolute view of time dilation and the latter used the operation of "checking clocks" to explain the relativity view of time dilation.

The theory of ether raised by the scientists in the $19^{\text {th }}$ century meets some difficulties in explaining the phenomenon happening in a lot of experiments. But has the Relative Space-Time view passed through the tests of practice? Or, can it verify itself by this kind of tests? They are the subjects to be discussed as following.

\section{Analysis of Some Classical Experiments in Special Relativity}

We have to beware that the objects of Space-time experimental research not only are atomic clocks readings, but also include frequency of rays and average life of flying meson etc. So time dilation not only are the motion clock go more slowly, but also include the frequency of rays of motion source drop off and the average life of flying meson is extended etc.

According to the special relativity, Observers can only observe the rhythm of motion clock drop off, but in the experiments, not only people can observe the rhythm of motion clock drop off, but also can observe such two phenomena: the rhythm of motion clock goes up, and the rhythm of motion clock unchanged. The rhythm of motion clock drop off has been called the time dilation effect by people, here the author can only call the "phenomenon of the rhythm of motion clock goes up" time contraction effect, and "the phenomenon of the rhythm of motion clock unchanged" time equivalence effect. Following analysis will prove that in the experiment people observed what kind of effect is time dilation, time contraction or time equivalence? This depends on how they choose reference system.

The following the author will analyse some classical experiments in earth reference frame, in order to facilitate our discussion, the author is going to use three coordinate systems: $k, k^{\prime}$ and $k^{\prime \prime} . k$ frame is static, $k^{\prime}$ and $k^{\prime \prime}$ frame moving in the opposite direction at the same speed, let the speed of $k^{\prime}$ is $v$, and the speed of $k^{\prime \prime}$ is $-v$, these classical experiments can divided into three types:

Experimental type I: the "clock" that experimenters wanted to measure was in 
the $k^{\prime}$ frame, while experimenters (or measuring instruments) were in the $k$ frame, the relative velocity between $k$ and $k^{\prime}$ is $V$, the time dilation effect can be observed in experimental type I. We have to pay attention, when the time dilation effect was happening, the observers (or measuring instruments) can measure that the motion clock go more slowly, and the frequency of rays of motion source drop off, or the average life of flying meson is extended, etc. Among them, the typical ones were:

1) In 1951, Lederman measured the meson $\pi^{-}$flying in an accelerator; in 1952, Durbin, in 1971, Ayres et al. measured meson $\pi^{ \pm}$flying in an accelerator; in 1959, Burrowes measured the mason $K^{ \pm}$flying in an accelerator. These experiments all proved the average life of flying meson was extended [3].

2) In 1968, Farley et al. measured the meson $\mu^{-}$flying in a cyclotron, the data of the experiment was $\beta \approx 0.996, \gamma \approx 12$, the experiment also proved the average life of flying meson was extended [3].

Though these experiments have shown us the time dilation effect, they were inadequate to prove the view of relative Space-Time is right! For:

1) The experiment type I measured time dilation in $K^{\prime}$ from $K$, but not from $K^{\prime}$ to get the same conclusion in $\mathrm{K}$. So, we cannot say that the view of relative Space-Time is correct.

2) According to the special relativity, people want to observe the relativity of time dilation, the premise is that there must be a "clock checking" operation between the two relative motion inertial frames, but the "checking clocks" operation has not feasibility in practice, so until today any experiment cannot prove that the relative space-time view is correct [4].

The time dilation of a moving clock should be found:

$$
\Delta t=\Delta t^{\prime} / \sqrt{1-v^{2} / c^{2}}
$$

Formula (1) proves that the time dilation is just related to the speed of clock, so how to correctly measure the movement speed $V$ of the clock and how to choose the coordinate system to measure the movement speed $V$ of the clock are all the problems we have to solve. If these problems cannot be solved, the changes in the rhythm of the moving clock cannot be correctly calculated either. The third part of this paper will discuss these problems in depth.

Experimental type II: the "clock" that experimenters wanted to measure was in the $k$ frame, while experimenters (or measuring instruments) were in $k^{\prime}$ frame, the relative velocity between $k$ and $k^{\prime}$ is $V$, the time contraction effect can be observed in experimental type II. We have to pay attention, when the time contraction effect was happening, the observers(or measuring instruments ) can measure that the motion clock go more quickly, and the frequency of rays of motion source goes up, or the average life of flying meson is shortened, etc. With the help of Mossbauer effect, the experiment by Hay and his partners in 1960 to verify the transverse Doppler shift can be regarded as experiment type II [3].

Doppler shifts are caused by relative motion, Hay's experiment is shown in Figure 1. The experimental device composed of such two parts: $\gamma$ source $C_{o}^{57}$ 


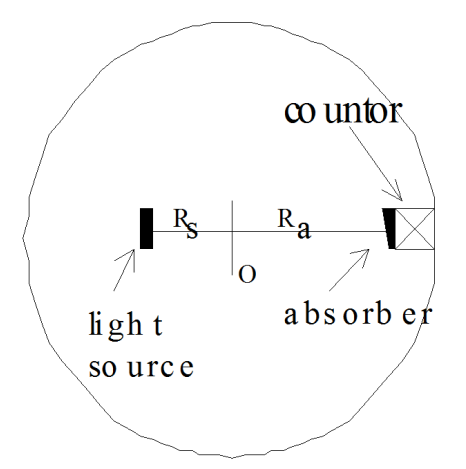

Figure 1. The sketch map of Hay's experiment.

and $\gamma$-ray receiver, while the $\gamma$-ray receiver consisted of the counter and absorber $\mathrm{Fe}^{57}$. Whole experimental device are all placed on a disk, point $O$ is the centre of the disc.

The $\gamma$ source and the centre of disk is very close, the distance between source and point $O$ is $R_{s}$; the $\gamma$-ray receiver is in the edge of the disc, the distance between receiver and point $O$ is $R_{a}$. When the disc is rest, the relative speed between the $\gamma$ source and the receiver is zero; When the disk rotated at an angular velocity of $\omega$, the linear speed of the $\gamma$ source was $V_{s}=\omega R_{s}$, the linear speed of the receiver was $V_{a}=\omega R_{a}$, the relative speed between the receiver and the source was $\omega\left(R_{a}+R_{s}\right)$, so the receiver can be detect the transverse Doppler shift of $\gamma$-rays. In the laboratory coordinate system, Hay and his partners calculated the Doppler shift of $\gamma$-rays:

$$
\begin{aligned}
& f_{s}=f / \sqrt{1-V_{s}^{2} / c^{2}} \\
& f_{a}=f / \sqrt{1-V_{a}^{2} / c^{2}}
\end{aligned}
$$

In the laboratory coordinate system, the author explain the physical meaning of formula (2) and formula (3): $f$ is the frequency of the $\gamma$ rays measured by the receiver when the $\gamma$ source and the receiver are relatively stationary, $f_{s}$ is the frequency of the $\gamma$ rays measured by the receiver when the relative speed between the $\gamma$ source and the receiver is $V_{s}, f_{a}$ is the frequency of the $\gamma$ rays measured by the receiver when the relative speed between the $\gamma$ source and the receiver is $V_{a}$, from formula (2) and formula (3), we get:

$$
\frac{v_{a}-v_{s}}{v_{s}}=\sqrt{\frac{1-V_{s}^{2} / c^{2}}{1-V_{a}^{2} / c^{2}}}-1 \approx \frac{1}{2 c^{2}}\left(V_{a}^{2}-V_{s}^{2}\right)=\frac{\omega^{2}}{2 c^{2}}\left(R_{a}^{2}-R_{s}^{2}\right)
$$

In this experiment, Hay and his partners put the $\gamma$ source at the centre of the disk $\left(R_{s}=0\right)$, the receiver was put at the edge of the disk (where $R_{a} \approx 6.2 \mathrm{~cm}$ ). The angular velocity of the disk is 500 circles per second, the error between the calculated value and the experimental result was $2 \%$.

With a careful analysis, we find the way of doing the experiment by Hay violated the special relativity. In the special relativity, the formula of transverse Doppler shift formula is expressed as follows [5]:

$$
f^{\prime}=f \sqrt{1-V^{2} / c^{2}}
$$


where $V$ is the relative speed between two frames. In the Hay's experiment, the relative speed between $\gamma$ source and the receiver is $\omega R_{a}$, According to special relativity, the transverse Doppler shift in the experiment is:

$$
f^{\prime}=f \sqrt{1-\left(\omega R_{a}\right)^{2} / c^{2}}
$$

In formula (6), $f$ was the frequency of $\gamma$-rays measured by the receiver when the disc was static(the relative speed between source and receiver was zero), and $f^{\prime}$ was the frequency of $\gamma$-rays measured by the receiver when the disk was rotating at the uniform speed(the relative speed between source and receiver was $\omega R_{a}$ ). According to formula (6), we get $f^{\prime}<f$, which indicated the frequency of $\gamma$-rays drop off when the relative motion between the receiver and the $\gamma$ source occurred, this phenomenon was called "time dilation" by physicists; But according to formula (2), (3), we get: $f_{s}>f, f_{a}>f$, which indicated that the frequency of $\gamma$-rays goes up when the relative motion between the receiver and the $\gamma$ source was occurred, the author can only call such phenomenon "time contraction". It shows that the result of Hay's experiment was not "time dilation" but in the opposite, it just is "time contraction". That is, the observer found that the moving clock didn't go slowly, but quickly. There is not time contraction in the special relativity, Hay's experiment has denied the Space-time view of the Special relativity, without question.

Experiment type III: The "clock" that experimenters wanted to measure was in the $k^{\prime}$ frame (or $k^{\prime \prime}$ frame), while experimenters (or measuring instruments) were in the $k^{\prime \prime}$ frame (or $k^{\prime}$ frame), the relative velocity between $k^{\prime}$ and $k^{\prime \prime}$ is $2 V$, the time equivalence effect can be observed in experiment type III. We have to pay attention, when the time equivalence effect happens, the rhythm of the motion clock and the frequency of rays of motion source and the average life of flying meson remains unchanged. The experiment made by Champeny and Moon in 1961 can be regarded as experiment of type III [3].

What is difference to the experiment done by Hay is that Champency and Moon put $\gamma$ source $C_{o}^{57}$ and receiver at the edge of a rotating disk, that means, $R_{s}=R_{a}$ (Figure 1). So, the relative speed between the $\gamma$ source and the receiver is $2 \omega R_{a}$. According to the special relativity, the Doppler shift should be [5]:

$$
f^{\prime}=f \sqrt{1-\left(2 \omega R_{a}\right)^{2} / c^{2}}
$$

In formula (7), $f$ should be the frequency of $\gamma$-rays measured when the disk is rest, and $f^{\prime}$ should be the frequency of $\gamma$-rays measured when the disk is rotation, but the actual result was:

$$
f^{\prime}=f
$$

In the Champency's experiment, the relative speed between the receiver and the $\gamma$ source is $2 V$, but the receiver did not still detect any Doppler shift, the author can only call such phenomenon "time equivalence" effect. There is no time equivalence in the special relativity; Champency's experiment has denied the Space-time view of the Special relativity again. 
There are more similar experiments similar to prove above findings. Put two clocks in the same round track and make them fly in the opposite direction at the same speed. Though they have relative motion to each other, the reading is still the same [3]. Some physicists intended to reject the result of this experiment by that special relativity can only be applied in inertia frame [3]. Here the author point out that some experimenters have measured average life of meson $\mu^{-}$, $\pi^{ \pm}, K^{ \pm}$in accelerate (Cyclotron) frames, because time dilation was appeared, so many physicists agreed these experiments. But the experiment in which two clocks moving in the same round track at the same speed in the opposite direction just showed us the effect of "time equivalence" which cannot be explained by the special relativity. Therefore, many scientists adopted the conception that special relativity can only be applied with inertia frame so as to reject those experiments, they used different standard when judging experiments.

There is only time dilation effect in the special relativity, but there are three effects in these classic experiments: time dilation, time contraction and time equivalence, the facts have clearly indicated that the Space-time view of the Special relativity is wrong.

\section{Analysis and Discussion on the Problems}

The author divides the experiments into three types in researching the view of relative Space-Time. "Time dilation" effect was appeared in the experimental type I, "Time contraction" effect was appeared in experimental type II, "Time equivalence" effect was appeared in the experimental type III. Because the special relativity cannot explain the results of these experiments, therefore the author reinterpreted these experiments with a new point of view.

The author thought that the universe is made up of numerous local systems, and each local system can be composed of two or more objects. A local system consisting of two (or several) objects with periodic motion among themselves can be called a stable local system. For example, the solar system is a stable local system composed of the sun and the planets. The earth and a clock with periodic motion around the earth can also form a stable local system, time dilation is absolute in a stable local system. After a detailed analysis of the local system, the author draws the following conclusion:

In a stable local system composed of clocks and planets, a coordinate system that is still in the gravitational potential (or gravitational potential) is a superior coordinate system. Only when people use a superior coordinate system can correctly calculate the time dilation of the motion clock.

About the whole argument process of the above conclusion, the author has made a detailed description in the chapter 3 of Analysis of Relativity Theory published in 2016. In the chapter 5 and chapter 6 of this book, the author also points out that when analyzing the time dilation of a moving clock in the earth reference frame, the earth coordinate system is a superior coordinate system. In the use of this superior coordinate system we must pay attention to such three points: 
1) Under the low movement circumstances, in superior coordinate system we can only correctly calculate the rhythm changes of the back-and-forth motion clock (or the periodic movement of the clock), but cannot correctly calculate the rhythm changes of the unidirectional moving clock, the velocity $\mathrm{V}$ of clock is that is average velocity of the back-and-forth motion clock (or the periodic movement of the clock). After a careful analysis of the Hafele's experiment, the author has proved that this point of view is correct [6].

2) Under the high movement circumstances, for example in the measurement of the meson clock in the cosmic ray, as long as the measurement distance is very short and the flying speed of the meson is very fast-even close to the speed of light, we can think that the flying meson clock will go through the measuring distance in a short moment. In the moment, we can also approximately think that the moving distance of the earth is very close to zero so that the earth coordinate system can be regarded as an instantaneous stationary coordinate system. Through the analysis of some classical experiments, the author has found that when calculating the time dilation of unidirectional and high speed moving clock in the instantaneous stationary coordinate system, the results obtained still have very high precision.

3) Only when the experimenters use the superior coordinate system can observe the time dilation, otherwise they can only observe the time contraction and time equivalence.

Base on above viewpoints, the author has explained the three effects of time dilation, time contraction, and time equivalence very clearly in Analysis of Relativity Theory in 2016, especially the time compression effect in Hay's experiment.

The author pointed out if people want to observe the time dilation in Hay experiment, the experimenters must put the receiver at the centre of the disk while $\gamma$ source should be put at the edge of the disk, only in this way, Hay's experiment can be regarded as experiment type I, because time dilation effect can only happen in experiment type I. But Hay and his partners had just confused the position of the $\gamma$ source and receiver, they put the receiver at the edge of the disk and $\gamma$ source be put at the centre of the disk, thus their experiment can only be regarded as experiment type II, so the time compression effect was appeared in their experiment. Hay's experiment indicated that the time dilation are not relativistic, and then it haven't symmetric property. More information can be found in Analysis of Relativity Theory in 2016.

\section{A Brief Conclusion}

According to the special relativity, observers can only observe the rhythm of motion clock dropping off, but in some classical experiments, people can observe not only the rhythm of motion clock dropping off, but also such two phenomena: the rhythm of motion clock going up, and the rhythm of motion clock unchanged. Clearly these experiments have all proved that the space-time view of the special relativity is wrong. For this reason, the author has reinterpreted the 
results of these experiments with a new point of view, and has solved the contradiction between the space-time theory and the experiments.

\section{References}

[1] Zhu, J.D. (2003) Discussing the Fundament of the Experiment about the Special Relativity. Journal of Shanghai University of Electric Power, No. 3, 57.

[2] Einstein, A. (1979) Evolution of Physics. Zhou, Z.W., Trans., Shanghai Science and Technology Publishing Company, Shanghai, 119-135.

[3] Zhang, Y.C. (1979) Experiment Foundation of the Special Relativity. Science Publishing House, Beijing, 74-84.

[4] Zhu, J.D. (2016) Analysis of Relativity Theory. Dong Hua University Press, CO LTD, 187-195.

[5] Kittel, C., Knight, W.D. and Ruderman, M.A. (1980) Mechanics, Berkeley Physics Course Vol.1. Chen, B.Q., Trans., Science Publishing House, Beijing, 467.

[6] Zhu, J.D. (2013) Ways of Correctly Measuring Time Dilation in the Moving Reference Frame. Journal of Shanghai University of Electric Power, No. 5, 501.

Submit or recommend next manuscript to OALib Journal and we will provide best service for you:

- Publication frequency: Monthly

- 9 subject areas of science, technology and medicine

- Fair and rigorous peer-review system

- Fast publication process

- Article promotion in various social networking sites (LinkedIn, Facebook, Twitter, etc.)

- Maximum dissemination of your research work

Submit Your Paper Online: Click Here to Submit

Or Contact service@oalib.com 